\title{
THE NON-LOCAL CAUCHY PROBLEM FOR SEMILINEAR INTEGRODIFFERENTIAL EQUATIONS WITH DEVIATING ARGUMENT
}

\author{
K. BALACHANDRAN AND M. CHANDRASEKARAN \\ Department of Mathematics, Bharathiar University, \\ Coimbatore-641 046, Tamil Nadu, India
}

(Received 21 October 1998)

\begin{abstract}
The aim of this paper is to prove the existence and uniqueness of mild and classical solutions of the non-local Cauchy problem for a semilinear integrodifferential equation with deviating argument. The results are established by using the method of semigroups and the contraction mapping principle. The paper generalizes certain results of Lin and Liu.
\end{abstract}

Keywords: existence of solutions; resolvent operator; integrodifferential equation; deviating argument

AMS 2000 Mathematics subject classification: Primary 34K05; 34K30

\section{Introduction}

Using the method of semigroups and the Banach fixed-point theorem, Byszewski [9] proved the existence and uniqueness of mild, classical and strong solutions of the following non-local Cauchy problem,

$$
\begin{gathered}
u^{\prime}(t)+A u(t)=f(t, u(t)), \quad t \in\left(t_{0}, t_{0}+a\right], \\
u\left(t_{0}\right)+g\left(t_{1}, \ldots, t_{p}, u(\cdot)\right)=u_{0},
\end{gathered}
$$

where $-A$ is the infinitesimal generator of a strongly continuous semigroup $T(t), t>0$, on a Banach space $X, 0 \leqslant t_{0}<t_{1}<\cdots<t_{p} \leqslant t_{0}+a, a>0, u_{0} \in X, f:\left[t_{0}, t_{0}+a\right] \times X \rightarrow X$ and $g:\left[t_{0}, t_{0}+a\right]^{p} \times X \rightarrow X$ are given functions. Subsequently, Byszewski investigated the same type of problem for a different class of evolution equations in a Banach space [4-11]. Moreover, Corduneanu [12] and Gripenberg et al. [15] studied the problem for Volterra integral equations of various types using a semigroup approach. The solution of (1.1) with $u\left(t_{0}\right)=u_{0}$ can be written as $[\mathbf{1 8}]$ :

$$
u(t)=T\left(t-t_{0}\right) u_{0}+\int_{t_{0}}^{t} T(t-s) f(s, u(s)) \mathrm{d} s, \quad t_{0} \leqslant t \leqslant t_{0}+a .
$$

Byszewski [9] proved the existence and uniqueness of the solution of (1.1) and (1.2) by using a fixed-point argument. He also proved that the mild solutions are classical solutions when $f \in C^{1}\left(\left[t_{0}, t_{0}+a\right] \times X, X\right)$. 
Now, consider the classical heat equation for a material with memory [15]:

$$
\left.\begin{array}{rl}
q(t, x) & =-E u_{x}(t, x)-\int_{0}^{t} b(t-s) u_{x}(s, x) \mathrm{d} s, \\
u_{t}(t, x) & =-\frac{\partial}{\partial x} q(t, x)+f(t, x), \\
u(x, 0) & =u_{0}(x) .
\end{array}\right\}
$$

The first equation gives the heat flux and the second is the balance equation. Equation (1.4) can be written as

$$
u_{t}(t, x)=\frac{\partial^{2}}{\partial x^{2}}\left[u(t, x)+\int_{0}^{t} b(t-s) u(s, x) \mathrm{d} s\right]+f(t, x), \quad u(0, x)=u_{0}(x) .
$$

It is clear that if non-local condition (1.2) is introduced to (1.5), then it will have a better effect than the classical condition $u(0, x)=u_{0}(x)$, since condition (1.2) is usually more precise for physical measurements than classical ones $[\mathbf{2 - 8}, \mathbf{1 0}, \mathbf{1 1}, \mathbf{1 6}]$.

Lin and Liu $[\mathbf{1 7}]$ have studied semilinear integrodifferential equations with the nonlocal Cauchy problem

$$
\begin{gathered}
u^{\prime}(t)=A\left[u(t)+\int_{0}^{t} F(t-s) u(s) \mathrm{d} s\right]+f(t, u(t)), \quad 0 \leqslant t \leqslant T, \\
u(0)+g\left(t_{1}, \ldots, t_{p}, u\left(t_{1}\right), \ldots, u\left(t_{p}\right)\right)=u_{0},
\end{gathered}
$$

in a Banach space $X$ with $A$ the generator of a strongly continuous semigroup and $F(t)$ a bounded operator for $t \in[0, T] . f:[0, T] \times X \rightarrow X$ and $g:[0, T]^{p} \times X^{p} \rightarrow X$ are given functions.

The purpose of this paper is to extend the technique of Lin and Liu [17] to the following integrodifferential equation with a deviating argument and non-local condition of the form

$$
\begin{gathered}
u^{\prime}(t)=A\left[u(t)+\int_{0}^{t} Z(t-s) u(s) \mathrm{d} s\right]+f(t, u(\sigma(t))), \quad 0 \leqslant t \leqslant T, \\
u(0)+g\left(u\left(t_{1}\right), \ldots, u\left(t_{p}\right)\right)=u_{0},
\end{gathered}
$$

where $A$ generates a strongly continuous semigroup in a Banach space $X, Z(t)$ is a bounded operator for $t \in[0, T], \sigma:[0, T] \rightarrow[0, T]$ is a continuous function such that $\sigma(t) \leqslant t$ for all $t, f:[0, T] \times X \rightarrow X$ and $g: X^{p} \rightarrow X$ are given functions. Let $J=[0, T]$. Since equation (1.3) played a very important role in the study of (1.1) with $u\left(t_{0}\right)=u_{0}$, the corresponding formula for (1.6) and (1.7) is given by [17],

$$
u(t)=R(t)\left[u_{0}-g\left(u\left(t_{1}\right), \ldots, u\left(t_{p}\right)\right)\right]+\int_{0}^{t} R(t-s) f(s, u(\sigma(s))) \mathrm{d} s,
$$

where the semigroup $T(\cdot)$ in $(1.3)$ is replaced by the resolvent operator $R(\cdot)$, the counterpart of $T(\cdot)$ for integrodifferential equations. 
The existence and uniqueness of solutions via variations of constants formula and other properties of resolvent operators have been studied in $[\mathbf{1 3}, \mathbf{1 4}]$. We are able to use the techniques developed by Pazy [18], Byszewski [9] and Lin and Liu [17] to study the problem (1.6) and (1.7). The results generalize Theorems 3.2 and 3.3 in [17].

\section{Preliminaries}

We make the following assumptions:

(i) $A$ generates a strongly continuous semigroup in a Banach space $X$; and

(ii) $Z(t) \in B(X), 0 \leqslant t \leqslant T, Z(t): Y \rightarrow Y$ for $x(\cdot)$ continuous in $Y, A Z(\cdot) x(\cdot) \in$ $L^{1}([0, T], X)$. For $x \in X, Z^{\prime}(t) x$ is continuous in $t \in[0, T]$, where $B(X)$ is the space of all linear and bounded operators on $X$, and $Y$ is the Banach space formed from $D(A)$, the domain of $A$, endowed with the graph norm. We give the following definitions.

Definition 2.1 (see [17]). $R(\cdot)$ is a resolvent operator of (1.6) with $f \equiv 0$ if $R(t) \in$ $B(X)$ for $0 \leqslant t \leqslant T$ and satisfies

(1) $R(0)=I($ the identity operator on $X)$;

(2) for all $u \in X, R(t) u$ is continuous for $0 \leqslant t \leqslant T$; and

(3) $R(t) \in B(Y), 0 \leqslant t \leqslant T$. For $y \in Y, R(\cdot) y \in C^{1}([0, T], X) \cap C([0, T], Y)$ and

$$
\begin{aligned}
\frac{\mathrm{d} R(t)}{\mathrm{d} t} y & =A\left[R(t) y+\int_{0}^{t} Z(t-s) R(s) y \mathrm{~d} s\right] \\
& =R(t) A y+\int_{0}^{t} R(t-s) A Z(s) y \mathrm{~d} s, \quad 0 \leqslant t \leqslant T .
\end{aligned}
$$

Definition 2.2. $u\left(., u_{0}\right) \in C([0, T], X)$ is a mild solution of $(1.6)$ and (1.7) if it satisfies

$$
u(t)=R(t)\left[u_{0}-g\left(u\left(t_{1}\right), \ldots, u\left(t_{p}\right)\right)\right]+\int_{0}^{t} R(t-s) f(s, u(\sigma(s))) \mathrm{d} s .
$$

Definition 2.3. A classical solution of (1.6) and (1.7) is a function $u(\cdot) \in C([0, T], Y) \cap$ $C^{1}([0, T], X)$ which satisfies (1.6) and $(1.7)$ on $[0, T]$ and we denote it by $u\left(., u_{0}\right)$.

The existence and uniqueness of resolvent operators is guaranteed by the following theorem, whose proof can be found in [14].

Theorem 2.4. Let assumptions (i) and (ii) be satisfied. Then (1.6) with $f \equiv 0$ has a unique resolvent operator. 


\section{Existence of solutions}

Theorem 3.1. Assume that

(i) A generates a strongly continuous semigroup in a Banach space $X$ with norm $\|\cdot\|$ and $u_{0} \in X$;

(ii) $f: J \times X \rightarrow X$ is continuous in $t$ on $J$ and there exists a constant $L>0$ such that

$$
\left\|f\left(t, u_{1}\right)-f\left(t, u_{2}\right)\right\|_{X} \leqslant L\left\|u_{1}-u_{2}\right\|_{X}, \quad t \in J, \quad u_{1}, u_{2} \in X
$$

(iii) $\sigma: J \rightarrow J$ is differentiable and $\sigma(t) \leqslant t$ for all $t$;

(iv) $g: X^{p} \rightarrow X$ and there exists a constant $K>0$ such that

$$
\left\|g\left(u\left(t_{1}\right), \ldots, u\left(t_{p}\right)\right)-g\left(v\left(t_{1}\right), \ldots, v\left(t_{p}\right)\right)\right\|_{X} \leqslant K \sup _{t \in J}\|u(t)-v(t)\|_{X}
$$

(v) $M=\max _{t \in J}\|R(t)\|_{B(X)}, q=(M K+M L T)<1$;

then problem (1.6) and (1.7) has a unique mild solution on $J$.

Proof. Let us take $E=C(J, X)$.

Then define an operator $F: E \rightarrow E$ by

$$
(F v)(t)=R(t)\left[u_{0}-g\left(v\left(t_{1}\right), \ldots, v\left(t_{p}\right)\right)\right]+\int_{0}^{t} R(t-s) f(s, v(\sigma(s))) \mathrm{d} s, \quad t \in J .
$$

Now, for every $v_{1}, v_{2} \in E$ and $t \in J$, we have

$$
\begin{aligned}
\|\left(F v_{1}\right)(t)- & \left(F v_{2}\right)(t) \|_{X} \\
\leqslant & \|R(t)\|_{B(X)}\left\|g\left(v_{1}\left(t_{1}\right), \ldots, v_{1}\left(t_{p}\right)\right)-g\left(v_{2}\left(t_{1}\right), \ldots, v_{2}\left(t_{p}\right)\right)\right\|_{X} \\
& \quad+\int_{0}^{t}\|R(t-s)\|_{B(X)}\left\|f\left(s, v_{1}(\sigma(s))\right)-f\left(s, v_{2}(\sigma(s))\right)\right\|_{X} \mathrm{~d} s \\
\leqslant & M K \sup _{t \in J}\left\|v_{1}(t)-v_{2}(t)\right\|_{X}+M L \int_{0}^{t}\left\|v_{1}(\sigma(s))-v_{2}(\sigma(s))\right\|_{X} \mathrm{~d} s \\
\leqslant & M K \sup _{t \in J}\left\|v_{1}(t)-v_{2}(t)\right\|_{X}+M L T \sup _{t \in J}\left\|v_{1}(t)-v_{2}(t)\right\|_{X} \\
\leqslant & (M K+M L T) \sup _{t \in J}\left\|v_{1}(t)-v_{2}(t)\right\|_{X}, \quad v_{1}, v_{2} \in X, \quad t \in J \\
\leqslant & q \sup _{t \in J}\left\|v_{1}(t)-v_{2}(t)\right\|_{X}, \quad 0<q<1 .
\end{aligned}
$$

This shows that operator $F$ is a contraction on $E$. Applying Banach's fixed-point theorem we get a unique fixed-point for $F$ and this point is the mild solution of problem (1.6) and (1.7) on $J$. 
Remark 3.2. If the inequality in assumption (ii) holds only for $u, v$ in a small ball in $X$, as shown in $[\mathbf{9}]$, then similar conditions as those in $[\mathbf{9}]$ can be obtained here.

Next, we prove that the mild solutions are classical solutions when $f \in C^{1}(J \times X, X)$.

Theorem 3.3. Let assumptions (i)-(v) in Theorem 3.1 be satisfied and let $u(\cdot)$ be the unique mild solution of (1.6) and (1.7). Assume further that $u_{0} \in D(A), f \in C^{1}(J \times X, X)$ and $g: X^{p} \rightarrow D(A)$. Then $u(\cdot)$ is a unique classical solutions of (1.6) and (1.7).

Proof. Since all the assumptions of Theorem 3.1 are satisfied, then problem (1.6) and (1.7) possesses a unique mild solution which is denoted by $u(\cdot)$. We will show that $u(\cdot) \in C^{1}(J, X)$.

Next, we shall show that this mild solution is a classical solution of problem (1.6) and (1.7) on $J$.

To this end, let

$$
B(s)=\frac{\partial}{\partial u(\sigma(s))} f(s, u(\sigma(s))) \sigma^{\prime}(s), \quad s \in J
$$

and

$$
\begin{aligned}
k(t)=R(t) f(0, u(\sigma(0)))+A[R(t) & \left.u(0)+\int_{0}^{t} Z(t-s) R(s) u(0) \mathrm{d} s\right] \\
& +\int_{0}^{t} R(t-s) \frac{\partial}{\partial s} f(s, u(\sigma(s))) \mathrm{d} s, \quad t \in J .
\end{aligned}
$$

Note that $u(0) \in Y$, from Definition 2.1 and our assumptions, $k(\cdot) \in E$. Thus, the method used in Pazy [18, pp. 184-187] or in the proof of the Theorem 3.3 above can be applied here to show that the integral equation

$$
w(t)=k(t)+\int_{0}^{t} R(t-s) B(s) w(\sigma(s)) \mathrm{d} s, \quad t \in J
$$

has a unique solution $w(\cdot) \in E$ (see also [1] ). Moreover, from the assumptions we have

$$
f(s, u(\sigma(s+h)))-f(s, u(\sigma(s)))=B(s)[u(\sigma(s+h))-u(\sigma(s))]+w_{1}(s, h)
$$

and

$$
f(s+h, u(\sigma(s+h)))-f(s, u(\sigma(s+h)))=\frac{\partial}{\partial s} f(s, u(\sigma(s+h))) h+w_{2}(s, h),
$$

where

$$
h^{-1}\left\|w_{i}(s, h)\right\| \rightarrow 0, \quad \text { as } h \rightarrow 0,
$$

uniformly on $s \in J$ for $i=1,2$. 
Define

$$
w_{h}(t)=\frac{u(t+h)-u(t)}{h}-w(t)
$$

Then, from $(3.1),(3.2),(3.3),(3.7)$ and the fact that $u(\cdot)$ is a mild solution, we obtain

$$
\begin{aligned}
& w_{h}(t)=\left(h^{-1}[R(t+h) u(0)-R(t) u(0)]-A\left[R(t) u(0)+\int_{0}^{t} Z(t-s) R(s) u(0) \mathrm{d} s\right]\right) \\
& +\left(h^{-1}\left[\int_{0}^{t+h} R(t+h-s) f(s, u(\sigma(s))) \mathrm{d} s-\int_{0}^{t} R(t-s) f(s, u(\sigma(s))) \mathrm{d} s\right]\right) \\
& -\left[R(t) f(0, u(\sigma(0)))+\int_{0}^{t} R(t-s) \frac{\partial}{\partial s} f(s, u(\sigma(s))) \mathrm{d} s\right] \\
& -\int_{0}^{t} R(t-s) B(s) w(\sigma(s)) \mathrm{d} s \\
& =\left(h^{-1}[R(t+h) u(0)-R(t) u(0)]-A\left[R(t) u(0)+\int_{0}^{t} Z(t-s) R(s) u(0) \mathrm{d} s\right]\right) \\
& +\left[h^{-1} \int_{0}^{h} R(t+h-s) f(s, u(\sigma(s))) \mathrm{d} s-R(t) f(0, u(\sigma(0)))\right] \\
& +h^{-1}\left[\int_{0}^{t} R(t-s)\left[\frac{\partial}{\partial s} f(s, u(\sigma(s+h))) h+w_{2}(s, h)\right] \mathrm{d} s\right. \\
& +\int_{0}^{t} R(t-s)\left[\frac{\partial}{\partial u(\sigma(s))} f(s, u(\sigma(s))) \sigma^{\prime}(s)\right. \\
& \left.\left.\times[u(\sigma(s+h))-u(\sigma(s))]+w_{1}(s, h)\right] \mathrm{d} s\right] \\
& -\int_{0}^{t} R(t-s) \frac{\partial}{\partial s} f(s, u(\sigma(s))) \mathrm{d} s-\int_{0}^{t} R(t-s) B(s) w(\sigma(s)) \mathrm{d} s \\
& =\left(h^{-1}[R(t+h) u(0)-R(t) u(0)]-A\left[R(t) u(0)+\int_{0}^{t} Z(t-s) R(s) u(0) \mathrm{d} s\right]\right) \\
& +\left[h^{-1} \int_{0}^{h} R(t+h-s) f(s, u(\sigma(s))) \mathrm{d} s-R(t) f(0, u(\sigma(0)))\right] \\
& +h^{-1}\left[\int_{0}^{t} R(t-s)\left[w_{1}(s, h)+w_{2}(s, h)\right] \mathrm{d} s\right. \\
& \left.+\int_{0}^{t} R(t-s) \frac{\partial}{\partial s}[f(s, u(\sigma(s+h)))-f(s, u(\sigma(s)))] \mathrm{d} s\right] \\
& +\int_{0}^{t} R(t-s) \frac{\partial}{\partial u(\sigma(s))} f(s, u(\sigma(s))) \sigma^{\prime}(s) \\
& \times\left[\frac{u(\sigma(s+h))-u(\sigma(s))}{h}-w(\sigma(s))\right] \mathrm{d} s
\end{aligned}
$$




$$
\begin{aligned}
\left\|w_{h}(t)\right\| \leqslant \| & h^{-1}[R(t+h) u(0)-R(t) u(0)]-A\left[R(t) u(0)+\int_{0}^{t} Z(t-s) R(s) u(0) \mathrm{d} s\right] \| \\
& +\left\|h^{-1} \int_{0}^{h} R(t+h-s) f(s, u(\sigma(s))) \mathrm{d} s-R(t) f(0, u(\sigma(0)))\right\| \\
& +\left\|h^{-1} \int_{0}^{t} R(t-s)\left[w_{1}(s, h)+w_{2}(s, h)\right] \mathrm{d} s\right\| \\
& +\left\|h^{-1} \int_{0}^{t} R(t-s) \frac{\partial}{\partial s}[f(s, u(\sigma(s+h)))-f(s, u(\sigma(s)))] \mathrm{d} s\right\| \\
& +N \int_{0}^{t}\left\|w_{h}(\sigma(s)) \sigma^{\prime}(s)\right\| \mathrm{d} s,
\end{aligned}
$$

where

$$
N=\max _{t \in J}\left\|R(t-s) \frac{\partial}{\partial u(\sigma(s))} f(s, u(\sigma(s)))\right\|_{B(X)} .
$$

From the definition of resolvent operator and our assumptions, it is clear that the norm of each one of the four first terms on the right-hand side of (3.8) tends to zero as $h \rightarrow 0$. Therefore, we have

$$
\left\|w_{h}(t)\right\|_{X} \leqslant \epsilon(h)+N \int_{\sigma(0)}^{\sigma(t)}\left\|w_{h}(s)\right\|_{X} \mathrm{~d} s,
$$

and $\epsilon(h) \rightarrow 0$ as $h \rightarrow 0$.

From (3.9), it follows by Gronwall's inequality that

$$
\left\|w_{h}(t)\right\|_{X} \leqslant \epsilon(h) \mathrm{e}^{T N},
$$

and, therefore, $\left\|w_{h}(t)\right\|_{X} \rightarrow 0, h \rightarrow 0, t \in J$.

This implies that $u(t)$ is differentiable on $J$ and that $w(t)$ is the derivative of $u(t)$. Since $w \in E, u$ is continuously differentiable on $J$.

Finally, to show that $u$ is the classical solution of problem (1.6) and (1.7). Observe that, from the continuous differentiability of $u$ and $f \in C^{1}(J \times X, X), t \rightarrow f(t, u(\sigma(t)))$ is continuously differentiable on $J$. Therefore, the linear Cauchy problem [17, Theorem 2.5]

$$
v(0)=u_{0}-g\left(u\left(t_{1}\right), \ldots, u\left(t_{p}\right)\right)
$$

has a unique classical solution $v(\cdot)$ given by

$$
v(t)=R(t)\left[u_{0}-g\left(u\left(t_{1}\right), \ldots, u\left(t_{p}\right)\right)\right]+\int_{0}^{t} R(t-s) f(s, u(\sigma(s))) \mathrm{d} s .
$$

The right-hand side of $(3.10)$ is $u(t)$ since $u(\cdot)$ is the mild solution. So we have $v(t)=u(t)$, $t \in J$, and, hence, $u(\cdot)$ is the classical solutions of (1.6) and (1.7). Hence, the theorem is proved. 


\section{References}

1. K. Balachandran, On a Volterra integral equation with deviating arguments, J. Appl. Math. Stoch. Analysis 3 (1990), 263-266.

2. K. Balachandran And M. Chandrasekaran, Existence of solution of a delay differential equation with nonlocal condition, Indian J. Pure Appl. Math. 27 (1996), 443-449.

3. K. BALACHANDRAN AND S. Illamaran, Existence and uniqueness of mild and strong solutions of a semilinear evolution equation with nonlocal conditions, Indian J. Pure Appl. Math. 25 (1994), 411-418.

4. L. ByszeWsKI, Strong maximum principles for parabolic nonlinear problems with nonlocal inequalities together with integrals, J. Appl. Math. Stoch. Analysis 3 (1990), 65-79.

5. L. Byszewski, Existence and uniqueness of solutions of nonlocal problems for hyperbolic equation $u_{x t}=F\left(t, x, u, u_{x}\right)$, J. Appl. Math. Stoch. Analysis 3 (1990), 163-168.

6. L. Byszewski, Theorems about the existence and uniqueness of continuous solution of nonlocal problem for nonlinear hyperbolic equation, Appl. Analysis 40 (1991), 173-180.

7. L. BYSZEWSKI, Strong maximum principles for parabolic nonlinear problems with nonlocal inequalities together with arbitrary functions, J. Math. Analysis Appl. 156 (1991), 457-470.

8. L. Byszewski, Uniqueness criterion for solution to abstract nonlocal Cauchy problem, $J$. Appl. Math. Stoch. Analysis 162 (1991), 49-54.

9. L. Byszewski, Theorems about the existence and uniqueness of solutions of a semilinear evolution nonlocal Cauchy problem, J. Math. Analysis Appl. 162 (1992), 494-505.

10. L. Byszewski, Existence of approximate solution to abstract nonlocal Cauchy problem, J. Appl. Math. Stoch. Analysis 5 (1992), 363-374.

11. L. Byszewski And V. LAKShmikAntham, Theorem about the existence and uniqueness of a solution of a nonlocal abstract Cauchy problem in a Banach space, Appl. Analysis 40 (1990), 11-19.

12. C. Corduneanu, Integral equations and applications (Cambridge University Press, 1991).

13. R. Grimmer, Resolvent operators for integral equations in a Banach space, Trans. Am. Math. Soc. 273 (1982), 333-349.

14. R. GRIMmer AND J. LiU, Liapunov-Razumikhin methods for integrodifferential equations in Hilbert space, in Delay and differential equations, pp. 9-24 (World Scientific, London, 1992).

15. G. Gripenberg, S. O. London and O. Staffans, Volterra integral and functional equations (Cambridge University Press, 1990).

16. D. JACKSON, Existence and uniqueness of solutions to semilinear nonlocal parabolic equations, J. Math. Analysis Appl. 172 (1993), 256-265.

17. Y. Lin AND J. H. LiU, Semilinear integrodifferential equations with nonlocal Cauchy problem, Nonlinear Analysis, Theory, Method Appl. 26 (1996), 1023-1033.

18. A. PAZY, Semigroups of linear operators and applications to partial differential equations (Springer, New York, 1983). 